\title{
CLINICAL OUTCOME OF RESPIRATORY VIRAL INFECTIONS IN CHILDREN UNDERGOING HEMATOPOIETIC STEM CELL TRANSPLANT
}

Torres JP1 ${ }^{1}$ ZUÑIGA Mำ, CORTÉS D ${ }^{1}$, CATALÁN P², VALENZUELA R ${ }^{1}$.

${ }^{1}$ Department of Pediatrics, ${ }^{2}$ Hospital Dr. Luis Calvo Mackenna, Faculty of Medicine, Universidad de Chile, Santiago, Chile.

\section{BACKGROUND}

There are few data regarding the clinical presentation of respiratory viral infections (RVI) in children undergoing hematopoietic stem cell transplant (HSCT), especially in developing countries.

\section{OBJECTIVE}

To determine the clinical outcome of RVI in children undergoing HSCT

\section{METHODS}

Prospective study in children $\leq 18$ years with cancer and HSCT admitted with fever at the Bone Marrow Transplant Unit. Hospital Calvo Mackenna, Santiago, Chile.

April 2016- March 2018

\section{Evaluation at admission:}

- Clinical examination - Laboratory tests

- Blood cultures -Nasopharyngeal sample for multiplex PCR (20 respiratory pathogens by Filmarray Respiratory Panel ())

Informed consent and assent

\section{Outcomes}

Respiratory virus detected Upper and Lower respiratory tract disease

- Oxygen supplementation Admission to the PICU

- Mechanical ventilatory support Days of fever Days of hospitalization Mortality Antimicrobial withdrawal

RESULTS (From a total of 45 episodes of fever, 30 episodes (67\%) were RVI (+) in 22 children) (Tables shows data from the 30 RVI (+) episodes).

\section{Variable}

Age in years (median)

Male gender ( $\mathrm{n} ; \%)$

Type of cancer $(n ; \%)$

Leukemia

Non lymphoblastic leukemia

Leukemia relapse

Others

Days after HSTC (median)

\section{9,5}

$22(73)$

$13(44)$

7 (22)

8 (27)

2 (7)

145

\begin{tabular}{lc}
\hline Respiratory virus & $\mathbf{n}(\%)$ \\
\hline Rhinovirus & $17(47)$ \\
Coronavirus & $7(20)$ \\
Parainfluenza & $4(11)$ \\
Respiratory & \\
syncytial virus & $3(8)$ \\
Adenovirus & $3(8)$ \\
Influenza & $2(6)$ \\
Single RVI & $25(83)$ \\
\hline Multiple RVI & $5(17)$ \\
\hline Table 2. Respiratory viruses detected.
\end{tabular}

\begin{tabular}{|c|c|}
\hline Variable & $n(\%)$ \\
\hline Any respiratory symptom & $20(67)$ \\
\hline Cough & $17(57)$ \\
\hline Rhinorrea & $14(47)$ \\
\hline Others & $6(20)$ \\
\hline $\begin{array}{l}\text { Median Absolute neutrophi } \\
\text { count }\left(\mathrm{mm}^{3}\right)\end{array}$ & 2407 \\
\hline $\begin{array}{l}\text { Median Absolute } \\
\text { lymphocyte count }\left(\mathrm{mm}^{3}\right)\end{array}$ & 805 \\
\hline Positive blood cultures & $1(3)$ \\
\hline
\end{tabular}

\section{Variable}

Upper respiratory infection

Lower respiratory infection

Oxygen supplementation

Admission to PICU

Mechanical ventilatory support

Days of fever after admission

Days of hospitalization

Mortality cases

Antimicrobial withdrawal

Table 4. Clinical outcome variables.

\section{CONCLUSIONS}

- RVI were frequently detected in fever episodes after HSCT in children (67\% of fever episodes were RVI (+).

- We observed a predominance of lower respiratory infection, oxygen requirement and long hospital-stay.

- Viral persistent-detection was reported in $22 \%$ of episodes. In $30 \%$ of episodes, antimicrobials were withdrawal after the RVI diagnosis.

- RVI detection might help to rationalize the use of antimicrobials in this population 